\title{
Terminator oligo blocking efficiently eliminates rRNA from Drosophila small RNA sequencing libraries
}

\section{Michelle L. Wickersheim and Justin P. Blumenstiel}

Department of Ecology and Evolutionary Biology, University of Kansas, Lawrence, KS

BioTechniques 55:269-272 (November 2013) doi 10.2144/000114102

Keywords: Drosophila; 2S rRNA; piRNA; miRNA; small RNA sequencing

Supplementary material for this article is available at www.BioTechniques.com/ article/114102.

A large number of methods are available to deplete ribosomal $R N A$ reads from high-throughput RNA sequencing experiments. Such methods are critical for sequencing Drosophila small RNAs between 20 and 30 nucleotides because size selection is not typically sufficient to exclude the highly abundant class of 30 nucleotide $2 S$ rRNA. Here we demonstrate that pre-annealing terminator oligos complimentary to Drosophila $2 S$ rRNA prior to 5' adapter ligation and reverse transcription efficiently depletes $2 S$ rRNA sequences from the sequencing reaction in a simple and inexpensive way. This depletion is highly specific and is achieved with minimal perturbation of miRNA and piRNA profiles.

Method Summary: Here we present a simple method for eliminating $2 S R N A$ from small RNA sequencing reactions in Drosophila with significant time and cost saving. This method may also have broader applications for other forms of RNA-seq.
High-throughput RNA sequencing (RNA-seq) has revolutionized our understanding of RNA biology, but the presence of high abundance RNA species that are not of direct interest can be problematic. For this reason, there is great interest in developing methods that enrich specific classes of RNA for RNA-seq applications. Historically, the most commonly used method for enriching mRNA depends on the presence of poly(A) tails. However, this method is not applicable in non-eukaryotes lacking polyadenylation; it is also incapable of enriching for many non-coding RNAs and degraded RNAs that lack poly(A) tails. In contrast to enriching for $\operatorname{poly}(\mathrm{A})$, an alternative approach is to directly deplete nuisance classes of RNA. A recent survey (1) compared different methods (2-6) for depleting nuisance species of rRNA, favoring rRNA hybridization to DNA oligos and removal of RNA-DNA hybrids by RNase $\mathrm{H}$ treatment.

For sequencing small RNAs (miRNAs, siRNAs and piRNAs), enrichment is usually achieved by size selection. Since these classes of RNA are typically between 20 and $30 \mathrm{nt}$, size selection from total RNA, followed by ligation, PCR, and further size selection, generally achieves good results (see Reference 7 for a review of small RNA sequencing methods). In Drosophila, however, size selection is not typically sufficient because the highly abundant class of $2 \mathrm{~S}$ rRNA is $30 \mathrm{nt}$ long. To deal with this problem, two approaches have been developed. One method, using RNase $\mathrm{H}$, employs hybridization with DNA oligos complementary to $2 \mathrm{~S}$ rRNA and directed degradation (8). A second method depletes $2 \mathrm{~S}$ rRNA by hybridization using oligos bound to magnetic beads, from which non-2S rRNA is removed off as supernatant (9). Both methods have proven effective in eliminating $2 \mathrm{~S}$ rRNA reads, but each requires additional steps and reagents which, in the case of magnetic beads, can be costly. We therefore wanted to determine if depletion could be achieved during 5' ligation and cDNA synthesis by specifically blocking 2S RNA products from jointly participating in these two reactions. For $2 \mathrm{~S}$ RNA, this is expected to yield truncated cDNA products lacking sequences complementary to the $5^{\prime}$ adapter. Without these sequences, library PCR amplification is impossible. Blocking with terminator oligos may thus serve as a simple means to eliminate $2 \mathrm{~S}$ rRNA from the sequencing reaction without additional enzymatic steps or use of magnetic beads.

To test this, we modified a previously published protocol (10) with the intent of precluding $5^{\prime}$ adapter ligation to $2 \mathrm{~S}$ rRNA. We first size selected for 19 to 30 nt RNAs with $15 \%$ acrylamide/urea gels, followed by $3^{\prime}$ adapter ligation. Prior to $5^{\prime}$ adapter ligation, we pre-annealed a $2 \mathrm{~S}$ rRNA

Table 1. 2S mapped reads from $2 \mathrm{~S}$ block (-) and (+) small RNA sequence runs. Percent genome mapping of total reads is in parentheses. The percentage of total reads in $2 \mathrm{~S}$ reads is also in parentheses.

\begin{tabular}{|c|c|c|c|c|}
\hline \multicolumn{2}{|c|}{ Experiment 1 } & \multicolumn{2}{c|}{ Experiment 2 } \\
\hline & 2S block (-) & 2S block (+) & 2S block (-) & 2S block (+) \\
\hline $\begin{array}{c}\text { Total reads } \\
\text { (\% mapped) }\end{array}$ & $919686(99)$ & $689333(98)$ & $1556883(98)$ & $1186377(98)$ \\
\hline Unique reads & 107102 & 154663 & 206277 & 221156 \\
\hline $\begin{array}{c}\text { 2S reads } \\
\text { \% total reads) }\end{array}$ & $515483(56)$ & $437(<0.1)$ & $454481(29)$ & $496(<0.1)$ \\
\hline
\end{tabular}




\section{Benchmark}

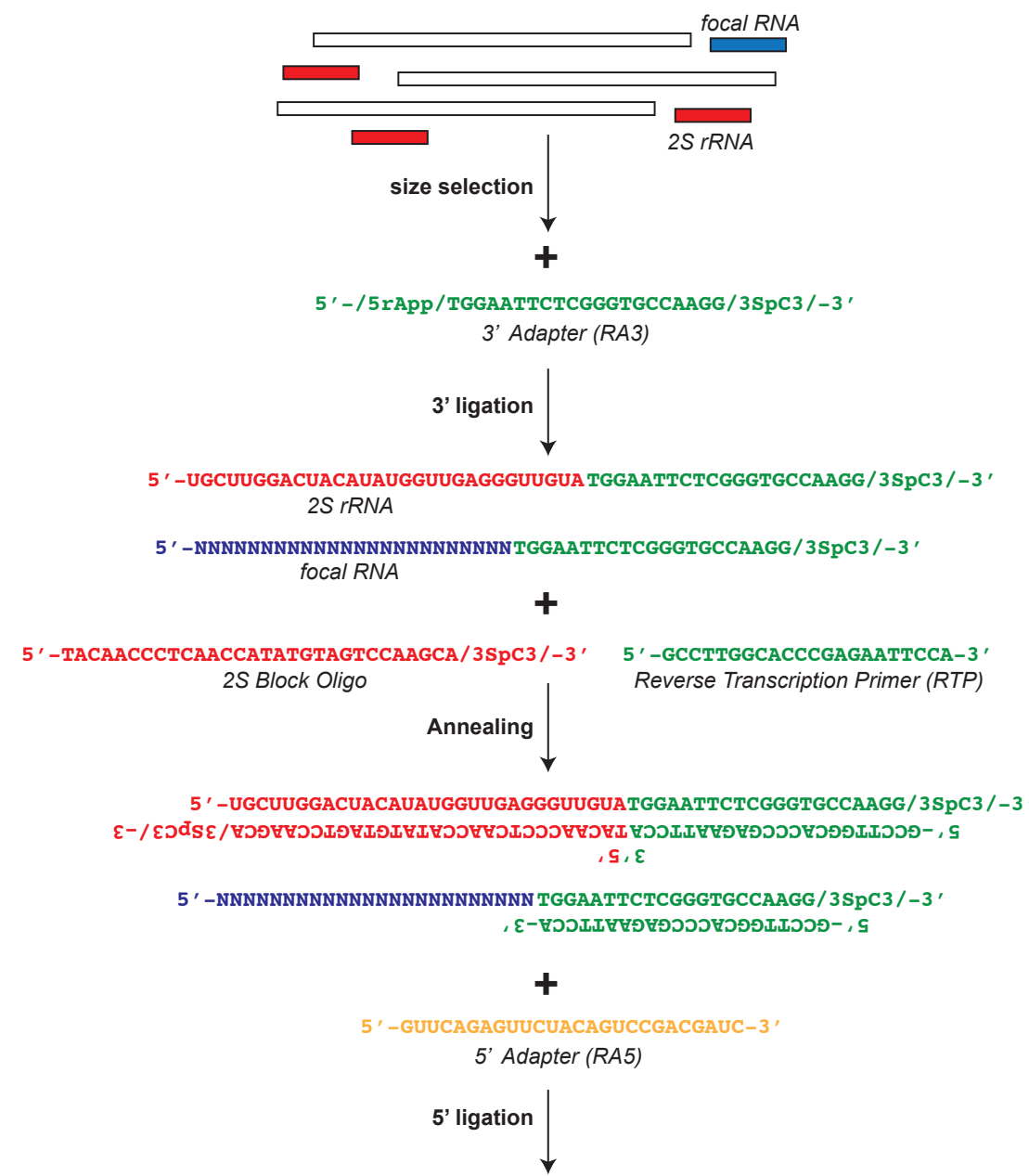

5' ligation block $\quad 5^{\prime}$-UGCUUGGACUACAUAUGGUUGAGGGUUGUATGGAATTCTCGGGTGCCAAGG/3SpC3/-3'

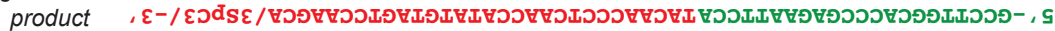
,,$\varepsilon$

5' -GUUCAGAGUUCUACAGUCCGACGAUCNNNNNNNNNNNNNNNNNNNNNNNN TGGAATTCTCGGGTGCCAAGG/3SpC3/-3' $\downarrow$

Reverse Transcription, PCR barcoding and sequencing

Figure 1. Overall scheme for small RNA library preparation with $2 \mathrm{~S}$ block reaction.

blocker complementary to the $2 \mathrm{~S}$ rRNA sequence and ending with a 3' $\mathrm{C} 3$ spacer (Figure 1). Subsequently, we annealed the reverse transcription (RT) primer complementary to the $3^{\prime}$ adapter. This RT primer was annealed prior to $5^{\prime}$ ligation to eliminate adapter-adapter ligation products (10). After these pre-annealing steps, 5' ligation and RT were done, followed by PCR-based barcode incorporation and
Illumina sequencing (see Supplementary Material). For these experiments, two separate size selected small RNA pools (experiment 1 and experiment 2) were extracted from Drosophila virilis ovaries. After gel purification and size selection, the two RNA pools were each split into no block and block treatments.

To measure depletion of $2 \mathrm{~S}$ rRNA, we quantified the proportion of 19 to 30 bp reads mapping to $2 \mathrm{~S}$ rRNA. Table 1 indicates that $2 \mathrm{~S}$ rRNA was nearly entirely eliminated when the $2 \mathrm{~S}$ block was utilized. Since the number of unique small RNA reads is greater for the blocked samples in both experiments, we can conclude that the block is not leading to PCR compression of library complexity. Examining the size distribution of reads (Figure 2), one can see that the $2 \mathrm{~S}$ block reaction largely eliminates the $30 \mathrm{bp}$ class of RNA without perturbing the underlying size distribution. In all cases, a $22 \mathrm{nt}$ peak of miRNA is evident against a background of presumptive piRNA and siRNA.

To determine whether non-specific $2 \mathrm{~S}$ block hybridization perturbed signatures of miRNA expression from ovaries, we mapped non-2S rRNA small RNA reads against the annotated miRNA library from D. virilis. Comparison of profiles was achieved by measuring the proportion of non- $2 \mathrm{~S}$ reads that mapped to these miRNAs. miRNA profiles were very similar between blocked and non-blocked libraries (Figure 3A,C). In experiment 1, there was some difference in the expression profile between two of the most highly expressed miRNAs. However, this was not the case in experiment 2 . To directly compare the miRNA profiles between experiments, we used Pearson's correlation coefficient $r$ as a metric (Figure 3C). Among all pairwise comparisons and considering all miRNAs, the pair with the most similar results were Experiment 2 (no 2S block) and Experiment 2 (2S block) $(r=0.999)$. Overall, results indicate that Experiment 1 (no 2S block) is an outlier since its Pearson's $r$ value is lower with all other samples. However, its miRNA profile is most similar to its own block treatment pair $(r=0.965)$. Since block pairs and no block pairs were never most similar to each other, we can conclude that blocking treatment did not result in a systematic bias in miRNA profile signatures. Excluding the four highly expressed RNAs, the most similar two pairwise results were achieved within RNA extraction rather than within block/no block treatment.

We also determined whether $2 \mathrm{~S}$ blocking perturbed the signature of small RNAs targeting transposable elements (TEs). We mapped non-2S/non-miRNA reads against 
Reads per Million
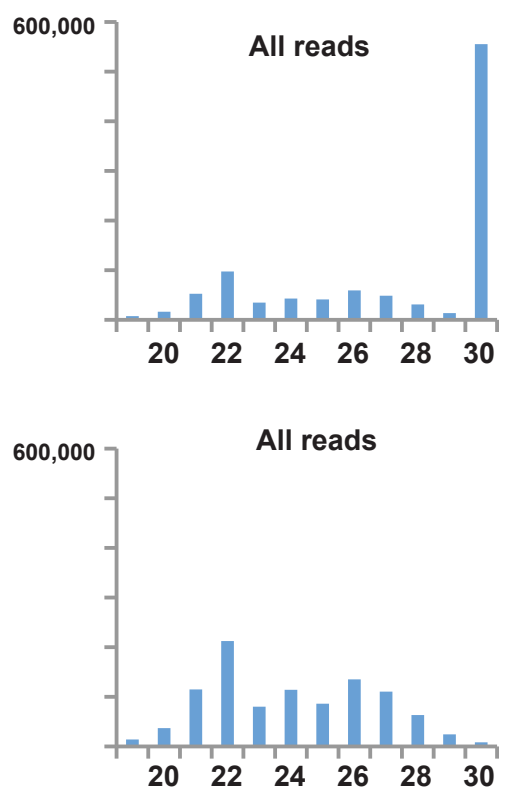

\section{Experiment 2}
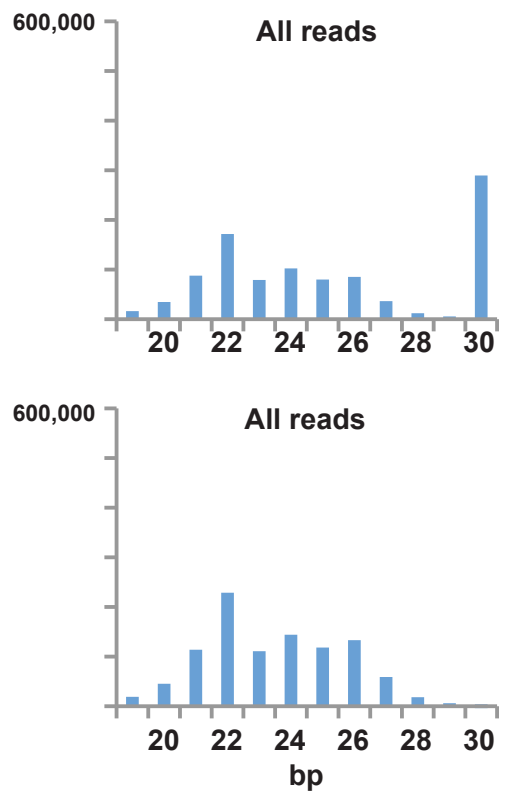

\section{Experiment 1}
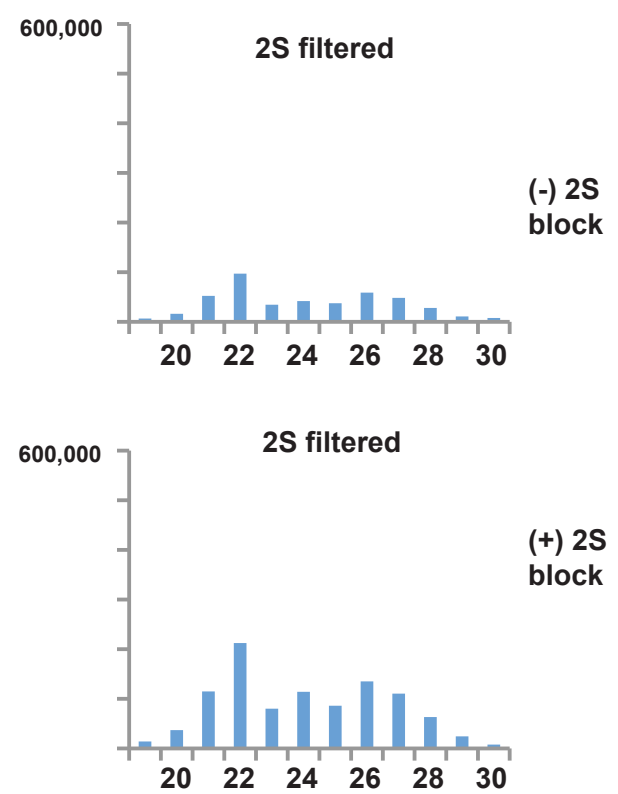

$(+) 2 S$

block

$(-) 2 S$

block
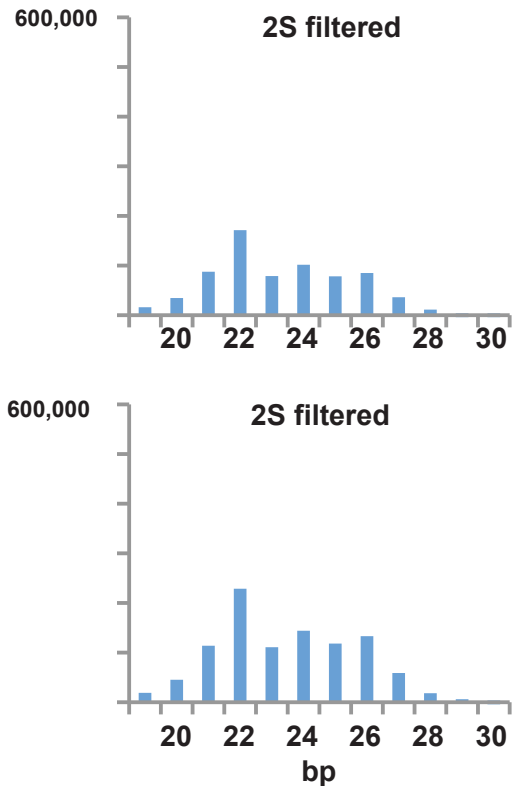

(+) $2 S$ block

Figure 2. Size distribution of small RNA reads from two experiments, with and without $2 \mathrm{~S}$ block treatment. $2 \mathrm{~S}$ filtered distributions were obtained after removal of $2 \mathrm{~S}$ mapping sequences. the 10 well annotated TEs from $D$. virilis. The vast majority of those mapping TEs were $23-30 \mathrm{nt}$ in length, indicating that they were piRNAs. TE mapping small RNA profiles are remarkably robust across all treatments and there is no evidence $2 \mathrm{~S}$ blocking perturbs these profiles (Figure 3B). Interestingly, small RNA profiles from TEs are more robust to experimental variation than miRNA profiles (Figure 3D). This might be explained by the fact that each TE represents many different piRNAs. In contrast to single miRNA measurements, pools of diverse piRNAs may be robust to stochastic variation in cloning, PCR and sequencing efficiency.

To conclude, we have demonstrated a simple and inexpensive method to limit rRNA reads from small RNA sequence reactions. This is most likely achieved by limiting $5^{\prime}$ adapter ligation to the double-stranded rRNA/oligonucleotide duplex. Previous studies have shown that 5' adapter/3' adapter ligation events are minimized in the presence of duplex DNA (10). The presence of a 3' C3 spacer may further contribute to ligation blocking. Since rRNA excess is a general problem for RNA-seq, similar blocking strategies that limit adapter ligation and full length cDNA synthesis may prove generally useful, limiting the need for magnetic beads and additional enzymatic steps.

\section{Author contributions}

MLW and JPB designed the experiments. MLW conducted the experiments. JPB wrote the paper.

\section{Acknowledgments}

Funding for this work was provided by NSF Award MCB-1022165 and NIH P20GM103638. This paper is subject to the NIH Public Access Policy

\section{Competing interests}

The authors declare no competing interests.

\section{References}

1. Adiconis, X., D. Borges-Rivera, R. Satija, D.S. Deluca, M.A. Busby, A.M. Berlin, A. Sivachenko, D.A. Thompson, et al. 2013. 


\section{Benchmark}

A.
Experiment 1

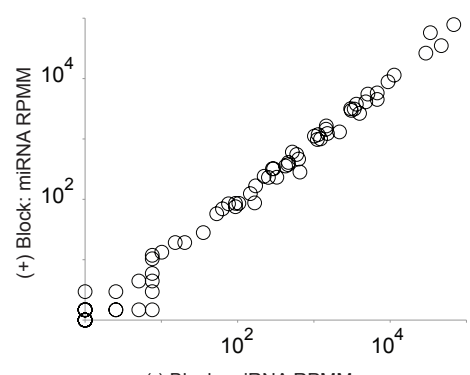

(-) Block: miRNA RPMM

B.

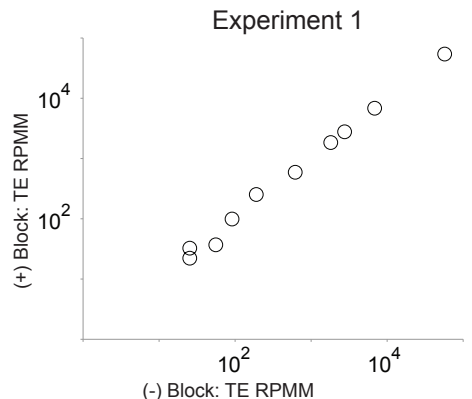

c.

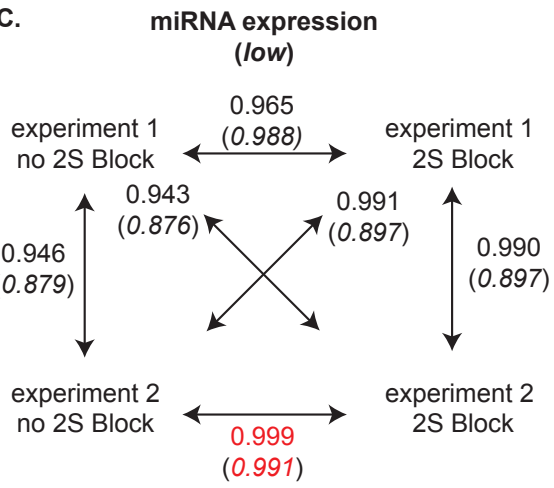

Experiment 2

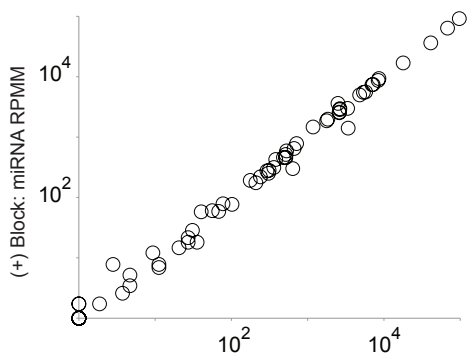

(-) Block: miRNA RPMM

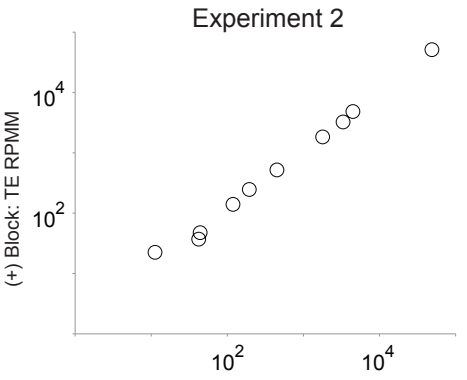

(-) Block: TE RPMM

D.

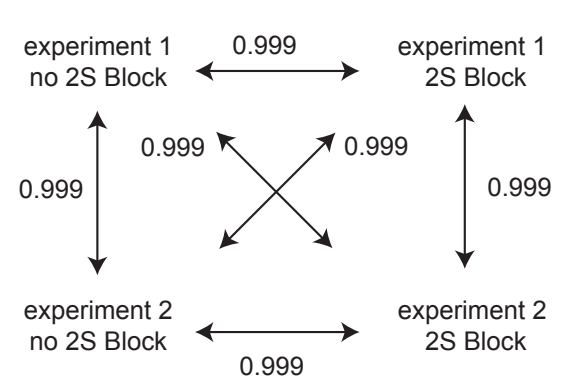

experiment 2 no $2 S$ Block

TE small RNA expression
Figure 3. Comparison of Drosophila virilis ovary small RNA profilesusing no block and $2 \mathrm{~S}$ block treatments. A) $\log 10-\log 10$ miRNA profiles from two experiments, comparing no block and $2 \mathrm{~S}$ block treatments, after $2 \mathrm{~S}$ rRNA sequence filtering. Measurements are in reads per million mapped (RPMM) where mapped (M) is measured from the total number of reads mapping to the D. virilis genome. B) $\log 10-\log 10$ transposable elements (TE) mapping small RNA profiles from two experiments, comparing non block and $2 \mathrm{~S}$ block treatments. Measurements are the RPMM of non-2S RNA/non-miRNA mapping reads that map to each of the annotated $D$. virilis TEs. C) miRNA profile similarity comparison as measured by Pearson's $r$. Values in parentheses are with highly expressed miRNAs excluded. Red indicates most similar pair among a set of comparisons. D) TE small RNA profile similarity. All results, block or no block, are highly similar.
Comparative analysis of RNA sequencing methods for degraded or low-input samples. Nat. Methods 10:623-629.

2. Morlan, J.D., K. Qu, and D.V. Sinicropi. 2012. Selective depletion of rRNA enables whole transcriptome profiling of archival fixed tissue. PLoS ONE 7:e42882.

3. Huang, R., M. Jaritz, P. Guenzl, I. Vlatkovic, A. Sommer, I.M. Tamir, H. Marks, T. Klampfl, et al. 2011. An RNA-Seq strategy to detect the complete coding and non-coding transcriptome including full-length imprinted macro ncRNAs. PLoS ONE 6:e27288.

4. Yi, H., Y.J. Cho, S. Won, J.E. Lee, H. Jin Yu, S. Kim, G.P. Schroth, S. Luo, and J. Chun. 2011. Duplex-specific nuclease efficiently removes rRNA for prokaryotic RNA-seq. Nucleic Acids Res. 39:e140.

5. Ramsköld, D., S. Luo, Y.C. Wang, R. Li, Q. Deng, O.R. Faridani, G.A. Daniels, I. Khrebtukova, et al. 2012. Full-length mRNA-Seq from single-cell levels of RNA and individual circulating tumor cells. Nat. Biotechnol. 30:777-782.

6. Tariq, M.A., H.J. Kim, O. Jejelowo, and N. Pourmand. 2011. Whole-transcriptome RNAseq analysis from minute amount of total RNA. Nucleic Acids Res. 39:e120.

7. Zhuang, F., R.T. Fuchs, and G.B. Robb. 2012. Small RNA expression profiling by high-throughput sequencing: implications of enzymatic manipulation. J. Nucleic Acids 2012:360358

8. Li, C., V.V. Vagin, S.H. Lee, J. Xu, S.M. Ma, H.L. Xi, H. Seitz, M.D. Horwich, et al. 2009. Collapse of Germline piRNAs in the Absence of Argonaute3 Reveals Somatic piRNAs in Flies. Cell 137:509-521.

9. Seitz, H., M. Ghildiyal, and P.D. Zamore. 2008. Argonaute loading improves the 5' precision of both MicroRNAs and their miRNA* strands in flies. Curr. Biol. 18:147-151.

10. Vigneault, F., D. Ter-Ovanesyan, S. Alon, and S. Eminaga. C.C. D, J.G. Seidman, E. Eisenberg, and M.C. G. 2012. High-throughput multiplex sequencing of miRNA. Curr Protoc Hum Genet Chapter 11:Unit 11 12 11-10.

Received 25 July 2013; accepted 06 September 2013.

Address correspondence to Justin P. Blumenstiel, Department of Ecology \& Evolutionary Biology, University of Kansas, Lawrence, KS. E-mail: jblumens@ku.edu

To purchase reprints of this article, contact: biotechniques@fosterprinting.com 